\title{
NEW ECLIPSING BINARY TIMES OF MINIMA IN THE NORTHERN SKY MEASURED DURING THE YEAR 2020
}

\author{
AUER, R.F. ${ }^{1,2}$
}

1) South-Moravian-Observatory, Chudcice 273, 66471 Veverská Bítýška, Czech Republic, auer.reinhold@gmail.com 2) Toscana-Remote-Observatory, Via Mulini a Vento, 9, Orciatico PI, Italy

Abstract: We present 167 times of minima of 69 eclipsing binaries in the Northern Sky determined from differential, partly multicolor photometry, during the year 2020.

\section{Introduction}

In 2020, during 203 observation nights, 393 objects were observed, 357 of these are eclipsing binaries. We registered 167 times of minima of 69 eclipsing binary systems in the Northern Sky during the year 2020. All observations were done with a CCD detector, using Johnson-Cousins filter set BVRcIc and filter Clear.

\section{Observation}

The private observatory SMO (South-Moravian-Observatory ${ }^{1}$ ) is located near Brno, Czech Republic (N 49.28171, $\mathrm{E} 16.45352^{\circ}$, altitude $243 \mathrm{~m}$ ). The main instrument is a $0.3 \mathrm{~m}\left(12^{\circ}\right)$ f/4 Newtonian telescope with a CCD camera G2-1600 from Moravian Instruments Inc. ${ }^{2}$, chip Kodak KAF1602 ${ }^{3}(1536 \times 1024$ px, size $9 \mu \mathrm{m})$ with a field of view (FOV) of 58.6'x 39.1' and Johnson-Cousins BVRcIc filters. The angular resolution is $2.29^{\prime \prime} / \mathrm{px}$.

The private observatory TRO (Toscana-Remote-Observatory) is located near Orciatico, Tuscany, Italy (N $43.42888^{\circ}, \mathrm{E} 10.71722^{\circ}$, altitude $\left.434 \mathrm{~m}\right)$. Its main instrument is a $0.3 \mathrm{~m}$ $\left(12^{\circ}\right) \mathrm{f} / 3.85$ Newtonian telescope with a CCD camera G2-1600 from Moravian Instruments Inc., chip Kodak KAF1602 (1536x1024 px, size $9 \mu \mathrm{m})$ with a FOV of 41.8'x 27.8' and Johnson-Cousins UBVRcIc filters. The angular resolution is $1.71^{\prime \prime} / \mathrm{px}$.

Imaging and photometric data processing has been done with SIPS $^{4}$.

\footnotetext{
${ }^{1}$ http://south-moravian-observatory.jimdo.com/

${ }^{2}$ http://www.gxccd.com/

${ }^{3}$ https://www.datasheets360.com/part/detail/kaf-1603me/7148940108719137862/

${ }^{4}$ Scientific-Imaging-Data-Processing-Software by Moravian Instruments
} 


\section{Data analysis}

The data were calibrated with bias, dark frames and flat fields. The differential aperture photometry was performed on the calibrated image sets using the SIPS-Photometry-Tool. During this process SIPS performs also the astronometry for each image of the data set and registers all main paramters of the stars using the astronomical catalogues UCAC- $4^{5}$ or USNO-B1.0 ${ }^{6}$ catalogue unless the star were not included in the UCAC-4 catalogue.

The comparison (CMP) and check (CHK) stars for the differential photometry were selected according to their $(\mathrm{B}-\mathrm{V})$ and $(\mathrm{J}-\mathrm{K})$ color indices that can be compared with the indices of the variable star, as well as comparable magnitudes based on the UCAC-4 catalog data. The minima estimations were performed with SILICUPS ${ }^{7,8,9}$.

\section{Results}

Table 1: Times of Minima

\begin{tabular}{|c|c|c|c|c|c|c|}
\hline VAR & CONST & $\begin{array}{c}\text { VAR- } \\
\text { TYPE }^{10} \\
\end{array}$ & HJD & ERROR & MIN-TYPE & FILTER \\
\hline AH Cas & Cas & EA/SD & 2459127.488786 & 0.000237 & $P$ & $\mathrm{~V}$ \\
\hline $\mathrm{AH}$ Cas & Cas & $\mathrm{EA} / \mathrm{SD}$ & 2459127.488802 & 0.000238 & $\mathrm{P}$ & $\mathrm{R}$ \\
\hline ASASSN-V J003242.76+412307.0 & Aur & EW & 2459160.372413 & 0.007813 & $\mathrm{~S}$ & $\mathrm{~V}$ \\
\hline ASASSN-V J003242.76+412307.0 & Aur & EW & 2459160.374019 & 0.002334 & $\mathrm{~S}$ & $\mathrm{R}$ \\
\hline ASASSN-V J013410.10+711512.1 & And & EW & 2459127.399096 & 0.000892 & $\mathrm{P}$ & $\mathrm{V}$ \\
\hline ASASSN-V J013410.10+711512.1 & And & EW & 2459127.399693 & 0.000795 & $\mathrm{P}$ & $\mathrm{R}$ \\
\hline ASASSN-V J014802.06+553251.7 & Cas & EA & 2459163.405522 & 0.003036 & $\mathrm{P}$ & $\mathrm{R}$ \\
\hline ASASSN-V J014802.06+553251.7 & Cas & EA & 2459175.421416 & 0.026797 & $\mathrm{~S}$ & $\mathrm{R}$ \\
\hline ASASSN-V J014802.06+553251.7 & Cas & EA & 2459175.422647 & 0.004364 & $\mathrm{~S}$ & $\mathrm{~V}$ \\
\hline ASASSN-V J014802.06+553251.7 & Cas & EA & 2459175.422828 & 0.007503 & $S$ & $\mathrm{C}$ \\
\hline ASASSN-V J060633_11+281722.2 & Cas & EA & 2458855.410678 & 0.000661 & $\mathrm{~S}$ & $\mathrm{C}$ \\
\hline ASASSN-V J180737.64+693804.1 & Cas & SR & 2459061.523209 & 0.002537 & $\mathrm{P}$ & V \\
\hline ASASsn101135ab Aur & Aur & EA & 2459175.456888 & 0.002757 & $\mathrm{P}$ & $\mathrm{C}$ \\
\hline ASASsn101135ab Aur & Dra & EA & 2459176.389516 & 0.001824 & $\mathrm{~S}$ & $\mathrm{C}$ \\
\hline AW Cam & Cam & EB & 2458861.337914 & 0.004890 & $\mathrm{P}$ & $\mathrm{C}$ \\
\hline BK Peg & Peg & $\mathrm{EA} / \mathrm{D}$ & 2459177.401642 & 0.000614 & $\mathrm{P}$ & $\mathrm{V}$ \\
\hline CSS_J003227.2+411407 & And & EW & 2459160.308247 & 0.005284 & $S$ & V \\
\hline CSS_J003227.2+411407 & And & EW & 2459160.446513 & 0.006351 & $\mathrm{P}$ & $\mathrm{R}$ \\
\hline CSS_J003227.2+411407 & And & EW & 2459160.461503 & 0.010131 & NA & $\mathrm{V}$ \\
\hline CSS_J065209+380857 & Aur & EA & 2459175.461313 & 0.001208 & $\mathrm{P}$ & $\mathrm{C}$ \\
\hline CSS_J065249.1+381317 & Aur & EW & 2459175.560712 & 0.000898 & $\mathrm{P}$ & $\mathrm{C}$ \\
\hline CSS_J066350.8+382226 & Aur & EW & 2459175.429109 & 0.001809 & NA & $\mathrm{C}$ \\
\hline CSS_J066350.8+382226 & Aur & EW & 2459175.623364 & 0.012396 & NA & $\mathrm{C}$ \\
\hline CSS_J066350.8+382226 & Aur & EW & 2459175.623364 & 0.012396 & NA & $\mathrm{C}$ \\
\hline
\end{tabular}

\footnotetext{
${ }^{5}$ arXiv:1212.6182 [astro-ph.IM]

${ }^{6}$ http://www.nofs.navy.mil/data/FchPix/

${ }^{7}$ Simply Light Curve Processing System is part of the SIPS bundle

${ }^{8}$ Fitting based on ,cmpfit' code by S. Moshier and C. Markwardt (based on NINPACK-1 Last Square Fitting Library by B. Garbow, K. Hilstrom, J. More)

${ }^{9}$ Light curve phenomenological model based on Mikulasek (2015)

${ }^{10}$ Type of the variables according to the VSX (The International Variable Star Index)
} 


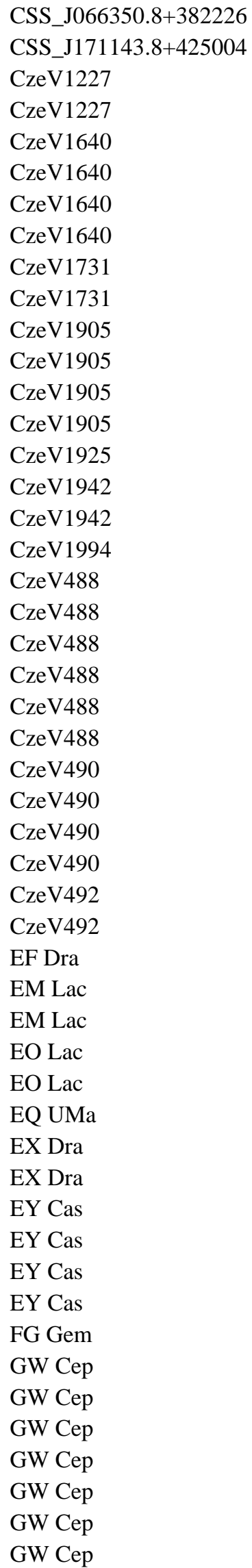

\begin{tabular}{|c|c|c|c|c|}
\hline Aur & EW & 2459175.623364 & 0.012396 & NA \\
\hline Her & EW & 2458987.492638 & 0.000638 & $\mathrm{P}$ \\
\hline Lac & EW & 2459108.507638 & 0.007352 & $\mathrm{P}$ \\
\hline Lac & EW & 2459108.509212 & 0.011412 & $\mathrm{P}$ \\
\hline Aur & EB & 2458857.469770 & 0.005766 & S \\
\hline Aur & EB & 2458857.472059 & 0.007908 & $\mathrm{~S}$ \\
\hline Aur & EB & 2458861.359933 & 0.008712 & S \\
\hline Aur & EB & 2458895.443231 & 0.000962 & $\mathrm{P}$ \\
\hline Dra & EA & 2459023.413498 & 0.001779 & $\mathrm{P}$ \\
\hline Dra & EA & 2459023.426090 & 0.001667 & $\mathrm{P}$ \\
\hline Aur & EB & 2458857.442553 & 0.000952 & $\mathrm{P}$ \\
\hline Aur & EB & 2458861.384172 & 0.001583 & $\mathrm{P}$ \\
\hline Aur & EB & 2458895.317817 & 0.002211 & $S$ \\
\hline Aur & EB & 2458902.321475 & 0.002103 & $\mathrm{~S}$ \\
\hline Aur & EB & 2458895.364834 & 0.002747 & S \\
\hline Aur & EB & 2458857.548612 & 0.017565 & $\mathrm{P}$ \\
\hline Aur & EB & 2458861.332716 & 0.001487 & $\mathrm{~S}$ \\
\hline Aur & $\mathrm{EB}$ & 2458857.256666 & 0.009884 & $\mathrm{NA}$ \\
\hline Cep & EB & 2459111.415122 & 0.001363 & NA \\
\hline Cep & EB & 2459111.418915 & 0.001182 & NA \\
\hline Cep & EB & 2459111.587825 & 0.001247 & NA \\
\hline Cep & EB & 2459111.589563 & 0.004680 & $\mathrm{NA}$ \\
\hline Cep & EB & 2459111.415122 & 0.001363 & NA \\
\hline Cep & EB & 2459111.418915 & 0.001182 & NA \\
\hline Cep & EW & 2459111.405975 & 0.001898 & NA \\
\hline Cep & EW & 2459111.405975 & 0.001898 & $\mathrm{P}$ \\
\hline Cep & EW & 2459111.416420 & 0.004332 & NA \\
\hline Cep & EW & 2459111.416420 & 0.004332 & $\mathrm{P}$ \\
\hline Cep & EA & 2459111.496434 & 0.009824 & $S$ \\
\hline Cep & EA & 2459111.516014 & 0.007714 & S \\
\hline Dra & EW/KW & 2459061.521893 & 0.000730 & S \\
\hline $\mathrm{Lac}$ & EW/KW & 2459088.444697 & 0.000460 & $\mathrm{P}$ \\
\hline Lac & EW/KW & 2459088.445856 & 0.000566 & $\mathrm{P}$ \\
\hline Lac & EA/SD & 2459088.444700 & 0.000399 & $\mathrm{P}$ \\
\hline Lac & EA/SD & 2459088.445858 & 0.000418 & $\mathrm{P}$ \\
\hline UMa & EW & 2458886.677739 & 0.010078 & $\mathrm{P}$ \\
\hline Dra & $\mathrm{UG}+\mathrm{E}$ & 2459097.397330 & 0.003506 & S \\
\hline Dra & $\mathrm{UG}+\mathrm{E}$ & 2459097.494360 & 0.000778 & $\mathrm{P}$ \\
\hline Cas & EW/KW & 2459115.359488 & 0.001559 & $S$ \\
\hline Cas & EW/KW & 2459115.360015 & 0.001401 & $\mathrm{~S}$ \\
\hline Cas & EW/KW & 2459115.597870 & 0.006024 & $\mathrm{P}$ \\
\hline Cas & $\mathrm{EW} / \mathrm{KW}$ & 2459115.601248 & 0.006315 & $\mathrm{P}$ \\
\hline Gem & EW & 2459197.489608 & 0.001478 & $\mathrm{P}$ \\
\hline Cep & $\mathrm{EW} / \mathrm{KW}$ & 2459098.339790 & 0.001850 & $\mathrm{~S}$ \\
\hline Cep & EW/KW & 2459098.348271 & 0.001494 & $\mathrm{~S}$ \\
\hline Cep & EW/KW & 2459098.497535 & 0.004668 & $\mathrm{P}$ \\
\hline Cep & $\mathrm{EW} / \mathrm{KW}$ & 2459098.500627 & 0.001996 & $\mathrm{P}$ \\
\hline Cep & EW/KW & 2459098.501757 & 0.003279 & $\mathrm{P}$ \\
\hline Cep & $\mathrm{EW} / \mathrm{KW}$ & 2459141.380975 & 0.000489 & NA \\
\hline Cep & EW/KW & 2459141.381781 & 0.000459 & NA \\
\hline
\end{tabular}


HS And

HS And

HT Cas

HT Cas

HT Cas

IP Peg

IP Peg

MNIC V20

MNIC V20

MW Lac

MW Lac

NSVS 260293

NSVS 49125 UMi

NSVS 49125 UMi

NSVS 49125 UMi

NSVS 6306374

NSVS 880674

NSVS 880674

NU UMa

NU UMa

NU UMa

NU UMa

PP Cam

PP Cam

PP Cam

PV Boo

PX And

PX And

QQ UMa

QQ UMa

ROTSE1 J153139.25+374359.0

ROTSE1 J153139.25+374359.0

ROTSE1 J161034.46+371538.9

ROTSE1 J161034.46+371538.9

SERIV 104

SERIV 104

SERIV 104

SERIV 104

SERIV 104

SERIV 104

SERIV 104

SERIV 104

SERIV 104

SERIV 104

SERIV 104

SERIV 104

TSVSC1 TN-N110200230-12-82-2

USNO B1 1382-0235440

USNO-B1.0 1478-0002611

USNO-B1.0 1478-0002611
And

And

Cas

Cas

Cas

Peg

Peg

Dra

Dra

Lac

Lac

Cep

UMi

UMi

UMi

And

Dra

Dra

UMa

UMa

UMa

UMa

Cam

Cam

Cam

Boo

And

And

UMa

UMa

$\mathrm{CrB}$

$\mathrm{CrB}$

$\mathrm{CrB}$

$\mathrm{CrB}$

Cas

Cas

Cas

Cas

Cas

Cas

Cas

Cas

Cas

Cas

Cas

Cas

Dra

UMa

Cas

Cas

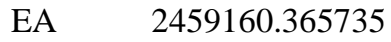

EA $\quad 2459160.365964$

UGSU+E $\quad 2459104.336647$

UGSU+E $\quad 2459104.404352$

UGSU+E 2459104.472452

UG+E 2459083.498770

UG+E 2459083.499148

EW 2458956.421430

EW 2458963.454338

EA/DM 2459108.400512

EA/DM 2459108.400708

EW 2459210.286058

E 2459174.562598

E 2459174.563525

E 2459174.564196

EW 2459111.410700

EA+DSCT 2458924.374809

EA+DSCT 2458924.591006

EA $\quad 2458908.333357$

EA 2458908.334235

EA 2458908.334263

EA 2458908.334729

EA 2459061.359046

EA 2459061.360231

EA 2459061.360716

EW 2458945.465061

$\mathrm{NL} / \mathrm{VY}+\mathrm{E} \quad 2459111.410060$

$\mathrm{NL} / \mathrm{VY}+\mathrm{E} \quad 2459111.500097$

EW 2458932.386191

EW 2458946.551630

EW

2459002.448681

2459002.449069

EW

2458973.395345

EW

2458973.540866

2459139.554418

2459139.555708

2459159.420394

2459174.372956

2459174.374016

2459174.374928

2459175.307414

2459175.310446

2459175.313033

2459175.540966

$\begin{array}{ll}\text { EB } & 2459175.541383 \\ \text { EB } & 2459175.546928\end{array}$

exopl 2458965.332631

EA/SD $\quad 2458926.515328$

EW 2459115.424952

EW 2459115.427541
0.000422

0.000286

0.001445

0.002245

0.004194

0.000224

0.000451

0.000033

0.000444

0.004786

0.009699

0.002836

0.002610

0.001460

0.001929

0.001788

0.000543

0.000404

0.000758

0.000610

0.000836

0.000913

0.003129

0.001390

0.002695

0.000326

0.001585

0.000376

0.000461

0.001475

0.000613

0.001339

0.000967

0.000449

0.003456

0.000869

0.008930

0.002409

0.005727

0.006813

0.002391

0.012272

0.002450

0.008515

0.004223

0.018883

0.004117

0.001469

0.001137

0.001133
P R

P V

S C

$\mathrm{S} \quad \mathrm{C}$

S C

P R

P V

S C

S C

P R

$\begin{array}{ll}\mathrm{P} & \mathrm{V}\end{array}$

NA V

P $\quad$ V

P R

P I

P $\quad$ C

P C

S C

P V

P B

P I

P $\quad$ R

P B

P R

P $\quad$ V

P C

S C

P C

S C

P C

P R

P V

P C

P C

S R

$\begin{array}{ll}\mathrm{S} & \mathrm{V}\end{array}$

$\begin{array}{ll}P & V\end{array}$

$\begin{array}{ll}\mathrm{P} & \mathrm{R}\end{array}$

$\begin{array}{ll}P & V\end{array}$

P $\quad \mathrm{C}$

P $\quad \mathrm{C}$

P V

P $\quad$ R

S V

S C

S R

P $\quad \mathrm{V}$

NA C

$\begin{array}{ll}\mathrm{P} & \mathrm{R}\end{array}$

P V




\begin{tabular}{|c|c|c|c|c|c|c|}
\hline UU And & And & EW & 2459172.397177 & 0.000698 & $\mathrm{P}$ & I \\
\hline UU And & And & EW & 2459172.397262 & 0.000665 & $\mathrm{P}$ & $\mathrm{R}$ \\
\hline UU And & And & EW & 2459172.397270 & 0.000409 & $\mathrm{P}$ & $\mathrm{V}$ \\
\hline UU Cam & Cam & EW & 2459061.349927 & 0.015724 & $\mathrm{P}$ & $\mathrm{R}$ \\
\hline UU Cam & Cam & EW & 2459061.351975 & 0.017969 & $\mathrm{P}$ & V \\
\hline UU Cam & Cam & EW & 2459061.354959 & 0.000650 & $P$ & B \\
\hline UU Cam & Cam & EW & 2459062.380073 & 0.001079 & S & B \\
\hline UU Cam & Cam & EW & 2459062.382098 & 0.006642 & $S$ & $\mathrm{R}$ \\
\hline UU Cam & Cam & EW & 2459062.382381 & 0.005276 & $S$ & $\mathrm{~V}$ \\
\hline V0474 Cam & Cam & EW & 2458894.607622 & 0.000195 & $S$ & $\mathrm{C}$ \\
\hline V0474 Cam & Cam & EW & 2459185.394677 & 0.000927 & $\mathrm{P}$ & $\mathrm{R}$ \\
\hline V0474 Cam & Cam & EW & 2459185.396825 & 0.003242 & S & B \\
\hline V0530 Cam & Cam & EW & 2459061.384849 & 0.003245 & $\mathrm{P}$ & $\mathrm{R}$ \\
\hline V0567 Dra & Dra & DSCTC & 2459061.520004 & 0.000824 & $S$ & V \\
\hline V0585 Dra & Dra & SRB & 2459023.409011 & 0.004360 & $\mathrm{P}$ & $\mathrm{R}$ \\
\hline V0698 Cep & Cep & EA & 2459111.531538 & 0.000683 & $\mathrm{P}$ & $\mathrm{V}$ \\
\hline V0698 Cep & Cep & EA & 2459111.531538 & 0.000683 & $\mathrm{P}$ & V \\
\hline V0698 Cep & Cep & EA & 2459111.531675 & 0.000689 & $\mathrm{P}$ & $\mathrm{R}$ \\
\hline V0698 Cep & Cep & EA & 2459111.531675 & 0.000689 & $\mathrm{P}$ & $\mathrm{R}$ \\
\hline V0722 Her & Her & $\mathrm{Ea}$ & 2458987.467827 & 0.009366 & NA & B \\
\hline V0796 Cep & Cep & EW & 2459141.436631 & 0.004716 & $\mathrm{P}$ & $\mathrm{R}$ \\
\hline V0796 Cep & Cep & EW & 2459141.441716 & 0.003159 & $\mathrm{P}$ & V \\
\hline V0919 Cep & Cep & $\mathrm{ZZO}$ & 2459159.281890 & 0.000674 & $\mathrm{P}$ & V \\
\hline V0919 Cep & And & $\mathrm{ZZO}$ & 2459159.282639 & 0.000894 & $\mathrm{P}$ & $\mathrm{R}$ \\
\hline V0919 Cep & Cep & $\mathrm{ZZO}$ & 2459159.281981 & 0.000863 & $\mathrm{P}$ & $\mathrm{V}$ \\
\hline V0919 Cep & And & $\mathrm{ZZO}$ & 2459159.281981 & 0.000863 & $\mathrm{P}$ & V \\
\hline V0919 Cep & Cep & $\mathrm{ZZO}$ & 2459159.282617 & 0.000925 & $\mathrm{P}$ & $\mathrm{R}$ \\
\hline V0919 Cep & Cep & $\mathrm{ZZO}$ & 2459159.282617 & 0.000925 & $\mathrm{P}$ & $\mathrm{R}$ \\
\hline V1260 Tau & Tau & EA & 2459177.479691 & 0.000554 & S & V \\
\hline V1312 Cas & Cas & $\mathrm{EA} / \mathrm{RS}$ & 2459104.365969 & 0.001177 & $\mathrm{P}$ & $\mathrm{C}$ \\
\hline V1324 Cas & Cas & EA & 2459127.429521 & 0.000746 & $\mathrm{P}$ & $\mathrm{R}$ \\
\hline V1324 Cas & Cas & EA & 2459127.429943 & 0.000866 & $\mathrm{P}$ & V \\
\hline WISE J013215.0+704856 & Cas & EW & 2459127.369879 & 0.001383 & $\mathrm{P}$ & $\mathrm{V}$ \\
\hline WISE J013215.0+704856 & Cas & EW & 2459127.372400 & 0.001311 & $\mathrm{P}$ & $\mathrm{R}$ \\
\hline WISE J192224.2+564857 & Dra & EW/EA & 2459023.405997 & 0.013418 & $\mathrm{P}$ & $\mathrm{R}$ \\
\hline WISE J192224.2+564857 & Dra & EW/EA & 2459023.408975 & 0.016364 & $\mathrm{P}$ & B \\
\hline WZ And & And & EB & 2459197.391573 & 0.000791 & $\mathrm{P}$ & V \\
\hline ZTF J0146571.07+552932.4 & Cas & EW & 2459159.552831 & 0.011216 & $\mathrm{P}$ & V \\
\hline ZTF J0146571.07+552932.4 & Cas & EW & 2459175.327444 & 0.003717 & $\mathrm{~S}$ & $\mathrm{~V}$ \\
\hline ZTF J0146571.07+552932.4 & Cas & EW & 2459175.347556 & 0.010355 & $\mathrm{~S}$ & $\mathrm{R}$ \\
\hline ZTF J0146571.07+552932.4 & Cas & EW & 2459175.361143 & 0.010120 & $\mathrm{~S}$ & $\mathrm{C}$ \\
\hline ZTF J0146571.07+552932.4 & Cas & EW & 2459175.508892 & 0.012220 & $\mathrm{P}$ & $\mathrm{R}$ \\
\hline ZTF J0146571.07+552932.4 & Cas & EW & 2459175.512698 & 0.008350 & $\mathrm{P}$ & $\mathrm{C}$ \\
\hline
\end{tabular}




\section{Conclusions and Summary}

The SIPS photometry tool optionally shows all variables registered in the VSX database within the field of view (FOV). Beside of the project stars of the NYX databases of the Astronomical Institute of Charles University a lot of bycatch stars were measured.

\section{Acknowledgements:}

This paper makes use of data from UCAC4 catalogue (Zacharias et al., 2012), and of the International Variable Star Index (VSX) database, operated at AAVSO, Cambridge, Massachusetts, USA. This research has made use of the VizieR catalogue access tool, CDS, Strasbourg, France (DOI: 10.26093/cds/vizier).

\section{References}

Brát, L., 2006, OEJV, 23, 55, 2006OEJV...23...55B

Mikulasek, Z., 2015, A\&A, 584, A8, 2015A\&A...584A...8M

Prša, A., \& Zwitter, T., 2005, ApJ, 628, 426, 2005ApJ...628..426P

Samus, N.N., GCVS 5.1, Astronomy Reports, 61, 1, 80-88. VizieR Online Data Catalog, B/GCVS, B/GCVS

Zacharias, N., Finch, C. T., Girard, T. M. et al. 2012, VizieR Online Data Catalog, I/322, $\underline{\mathrm{I} / 322 \mathrm{~A}}$ 